\title{
Aspectos legales de la instrumentación quirúrgica en Colombia
}

Legal aspects of surgical instrumentation in Colombia

Aspectos jurídicos da instrumentação cirúrgica na Colômbia

\section{Viviana Galán Camargo ${ }^{1}$}

Recibido: 4 de abril de 2020

Aprobado: 15 de mayo de 2020

Publicado: 13 de julio de 2020

Cómo citar este artículo:

Viviana Galán Camargo. Aspectos legales de la instrumentación quirúrgica en Colombia.

DIXI 32, julio-diciembre 2020, 1-14.

DOI: https://doi.org/10.16925/2357-5891.2020.02.03

Artículo de investigación. https://doi.org/10.16925/2357-5891.2020.02.03

1 Instrumentadora quirúrgica de la Universidad de Santander (UDES) sede Bucaramanga. Especialista en Docencia Superior Universitaria. Diplomada en Docencia Superior Universitaria. Docente de la Universidad Santiago de Cali y de la Fundación Universitaria del Área Andina sede Pereira.

Correo electrónico: vivi8204@hotmail.com 


\section{Resumen}

Tema y alcance: el artículo analiza cómo el instrumentador quirúrgico, dada su formación académica, es un profesional calificado para ofrecer en forma integral idoneidad y criterios claros en los variados procedimientos quirúrgicos de las diferentes especialidades, manejo de asepsia y antisepsia, y control de infección, actuando siempre con responsabilidad, con sentido humanístico y ético, y guardando el sigilo profesional.

Características: hoy se observa cómo el instrumentador quirúrgico es líder en la generación de información sobre el servicio de sala de cirugía, funcionamiento de centrales de esterilización con calidad y competitividad, a fin de administrar y emitir conceptos técnicos sobre equipos intrahospitalarios de su competencia o externos de casas comerciales que presten el servicio en este departamento.

Hallazgos: el desarrollo profesional de la instrumentación quirúrgica en Colombia ha sido referente para América Latina.

Conclusiones: el instrumentador quirúrgico debe dar un trato humano a cada paciente de acuerdo con una jerarquía razonablemente sustentada de sus necesidades biológicas, psicológicas, sociales y espirituales.

Palabras clave: bioética, Estado Social de Derecho, ética, instrumentador quirúrgico, principios, valores.

\section{Abstract}

Subject and scope: The article analyzes how the "instrumentador quirúrgico" (Surgical assistant specializing in surgical instruments), given his academic training, is a qualified professional capable of offering comprehensively suitable and clear criteria in the various surgical procedures of the different specialties, asepsis and antisepsis management, and infection control, always acting with responsibility, with a humanistic and ethical sense, and keeping professional secrecy.

Characteristics: Today we can see how the "instrumentador quirúrgico" is a leader in generating information about operating room service, the operation of sterilization plants with quality and competitiveness, in order to administer and issue technical concepts on intra-hospital equipment or externally to commercial establishments that provide similar services.

Findings: The professional development of surgical instrumentation in Colombia has been a benchmark for Latin America.

Conclusions: The "instrumentador quirúrgico" must treat each patient humanely according to a reasonably supported hierarchy of their biological, psychological, social and spiritual needs.

Keywords: bioethics, Social State of Law, ethics, surgical instrument, principles, values.

\section{Resumo}

Objecto e âmbito: 0 artigo analisa como o "instrumentador quirúrgico", dada a sua formação académica, é um profissional qualificado capaz de oferecer critérios claros e adequados nos vários procedimentos cirúrgicos das diferentes especialidades, assepsia e gestão da antissepsia e controle de infecção, agindo sempre com responsabilidade, sentido humanístico e ético, e mantendo o sigilo profissional.

Características: Hoje podemos constatar como o "instrumentador quirúrgico" é líder na geração de informação sobre o serviço de centro cirúrgico, a operação de centrais de esterilização com qualidade e competitividade, de forma a administrar e emitir conceitos técnicos sobre equipamentos intra-hospitalares ou externamente ao comercial estabelecimentos que prestam serviços semelhantes.

Resultados: 0 desenvolvimento profissional de instrumentação cirúrgica na Colômbia tem sido uma referência para a América Latina.

Conclusões: 0 "instrumentador quirúrgico" deve tratar cada paciente com humanidade de acordo com uma hierarquia razoavelmente sustentada das suas necessidades biológicas, psicológicas, sociais e espirituais.

Palavras-chave: bioética, Estado Social de Direito, ética, instrumento cirúrgico, princípios, valores. 


\title{
INTRODUCCIÓN
}

La Instrumentación Quirúrgica es una profesión del área de la salud en Colombia reglamentada por la Ley 784 de 2002, cuyo

\begin{abstract}
[...] ejercicio requiere título de idoneidad universitaria, basada en una formación científica, técnica y humanística, docente e investigativa y cuya función es la planeación, organización, dirección, ejecución supervisión y evolución de las actividades que competen al Instrumentador Quirúrgico Profesional como parte integral del equipo de salud?
\end{abstract}

El instrumentador brinda atención segura al paciente quirúrgico dentro y fuera del quirófano, mediante la aplicación de conocimientos científicos, técnicos y tecnológicos en intervenciones invasivas y no invasivas, procesos de esterilización, aplicación de normas universales de bioseguridad, salud pública, saneamiento ambiental, planeación, organización, administración del talento humano, del recurso físico y tecnológico, enmarcados dentro de las normas y principios éticos, y orientados hacia el servicio social. De igual forma, da asesoría y capacitación sobre el uso y manejo de equipos y dispositivos médicoquirúrgicos en los procedimientos de alta complejidad, mediante asistencia y soporte profesional directo.

\section{CARACTERÍSTICAS GENERALES}

El desarrollo profesional de la Instrumentación Quirúrgica en Colombia ha sido referente para América Latina. Por la autonomía universitaria contemplada en la Ley 30 de 1992, se inició en el país la formación profesional que marcó el desarrollo de otras competencias que hicieron de la Instrumentación Quirúrgica una profesión del área de las Ciencias de la Salud.

La Federación Latinoamericana de Instrumentadores Quirúrgicos (FLIQ), a la que pertenecen Chile, Argentina, Colombia, Bolivia, Uruguay y Brasil, ha contribuido a la integración comunitaria de intereses profesionales para construir un medio que posibilite afianzar una profesión poderosa y lograr una proyección de esta realidad hacia otros continentes. Profesionales colombianos han sido invitados a participar

1 Congreso de la República de Colombia. Ley 784 de 2002. Por medio de la cual se reforma la Ley 6 del 14 de enero de 1982 (M.P. José Gregorio Hernández; diciembre 23 de 2002). Do 45.046. 
en la creación de programas académicos como el que está en funcionamiento en la Universidad Nuestra Señora de La Paz, en La Paz, Bolivia.

En Colombia, la formación de los profesionales en Instrumentación Quirúrgica tiene una duración que ha variado entre diez y ocho semestres académicos, enmarcada dentro de las competencias relacionadas con el ser, el saber, el saber hacer y el saber comunicar, lo cual les permite desempeñarse en el área asistencial, centrales de esterilización, unidades de apoyo de diagnóstico y tratamiento, asesoría y comercialización de dispositivos y equipos médicoquirúrgicos, manejo de equipos de alta tecnología, rescate, proceso de conservación, embalaje y traslado de órganos.

\section{INSTRUMENTACIÓN QUIRÚRGICA EN COLOMBIA}

La instrumentación quirúrgica en Colombia inició hacia 1943 por la necesidad de personal capacitado para asistir a los médicos cirujanos durante el acto quirúrgico, en la preparación de equipos e insumos para cada una de las especialidades, y en la esterilización de ropa quirúrgica, instrumental y de dispositivos médicoquirúrgicos. La formación se estructuró inicialmente en nivel técnico y frente a los cambios vertiginosos de la cirugía, por el desarrollo de las tecnologías, evolucionó a nivel tecnológico; para entonces, los instrumentadores asumían responsabilidades con conocimientos acordes al momento histórico.

Desde la década de los setenta, las instrumentadoras quirúrgicas han sido quienes se han entrenado como perfusionistas cardiovasculares y han replicado sus conocimientos a otras instrumentadoras y a enfermeras. De igual manera, es también histórica la formación en microcirugía, lo que permitió el entrenamiento en este campo a médicos e instrumentadores quirúrgicos que laboran en grupos de trasplante de órganos.

La formación profesional se hizo necesaria por el vertiginoso desarrollo de la ciencia y la tecnología en la segunda mitad del siglo Xx. Fue así como hace más de veinte años inició en la Universidad de Boyacá el primer programa de formación profesional, seguido por otros en diferentes universidades del país. Actualmente, existen dieciséis programas de formación profesional.

Debido a que en el país ya no existe la formación técnica ni tecnológica en el área de la salud, se hizo necesario presentar al Congreso de la República un proyecto de ley que modificara la Ley 6 del 14 de enero de 1982 que reglamentaba el ejercicio técnico y tecnológico. 
Esta ley fue derogada por la Ley 784 del 23 de diciembre de 2002, que reglamenta el ejercicio de la instrumentación quirúrgica profesional, determina su naturaleza, propósitos y campos de aplicación, desarrolla los principios que la rigen y se señalan los entes de dirección, organización, acreditación y control de dicho ejercicio². El día nacional del instrumentador quirúrgico se celebra el 25 de octubre.

\section{ASPECTOS JURÍDICOS}

El instrumentador quirúrgico profesional deberá ser egresado de una facultad reconocida por el Estado colombiano y deberá aportar a su hoja de vida fotocopia del diploma y acta de grado de la identidad universitaria que certifique la terminación a cabalidad de los requisitos exigidos para su formación académica. Igualmente, deberá anexar fotocopia de registro profesional ante la Secretaría Salud nacional y departamental si fuera el caso. El instrumentador quirúrgico posee una formación universitaria para el ejercicio de la profesión en las diferentes aéreas, fundamentado en una formación científica, técnica, administrativa, humanística, docente e investigativa, orientada hacia la planeación, organización, dirección, ejecución, supervisión y evolución de las actividades que competen al instrumentador quirúrgico profesional como parte integral del equipo de salud. El instrumentador quirúrgico tiene a su cargo, entre otras actividades, la coordinación y manejo de centrales de esterilización, como también el manejo (montaje y cuidados) de equipos de alta tecnología (máquinas de perfusión, láser facoemulsificadores, ultrasonido, microscopio, endoscopios y autoclaves). ${ }^{3}$

El instrumentador quirúrgico, debido a su formación académica, es un profesional calificado para ofrecer en forma integral idoneidad y criterios claros en los variados procedimientos quirúrgicos de las diferentes especialidades y su especialidades quirúrgicas, manejo de asepsia y antisepsia, y control de infección, actuando siempre con responsabilidad, con sentido humanístico y ético, y guardando el sigilo profesional. Ser líder en la generación de información sobre el servicio de sala de cirugía, funcionamiento de centrales de esterilización con calidad y competitividad, y administrar

2 Ley 784 de 2002, artículo 1.

3 Id., artículo 2, parágrafo. 
y emitir conceptos técnicos sobre equipos intrahospitalarios de su competencia o externos de casas comerciales que presten el servicio en este departamento.

En Colombia, existe un marco jurídico para las profesiones del área de salud. Para instrumentación quirúrgica rigen las siguientes normas:

- Ley 784 del 23 de diciembre de 2002, por medio de la cual se reforma la Ley 6 del 14 de enero de 1982 y se reglamenta el ejercicio de la instrumentación quirúrgica profesional, se determina su naturaleza, propósitos y campos de aplicación, se desarrollan los principios que la rigen y se señalan los entes de dirección, organización, acreditación y control de dicho ejercicio.

- Resolución 2772 del 13 de noviembre de 2003, por medio de la cual se definen las características específicas de calidad para los programas de pregrado en ciencias de la salud.

- Ley 1438 del 19 de enero de 2011, por medio de la cual se reforma el Sistema General de la Seguridad Social en Salud y se dictan otras disposiciones.

- Ley 1164 del 3 de octubre de 2007, por medio del cual se dictan disposiciones en materia del talento humano en salud.

- Decreto 1295 del 20 de abril de 2010, por el cual se reglamenta el registro calificado de que trata la Ley 1188 de 2008 y la oferta y desarrollo de programas académicos de educación superior.

- Resolución 02183 del9 de julio de 2004, por la cual se adopta el Manual de Buenas Prácticas de Esterilización para Prestadores de Servicios de Salud.

- Resolución 06394 del 22 de diciembre de 2004, por medio de la cual se adopta el manual de procedimientos del sistema de cadena de custodia para el sistema penal acusatorio "7.11 FGN-CC-MEMPPIPS: manejo de los elementos materia de prueba o evidencias provenientes de entidades prestadoras de servicios de salud públicas o privadas"

- Circular000076del21denoviembrede2005. AplicaciónLey784de2002. Instrumentador Quirúrgico Profesional.

- Decreto 4725 del 26 de diciembre de 2005, por el cual se reglamenta el régimen de registros sanitarios, permiso de comercialización y vigilancia sanitaria de los dispositivos médicos para uso humano.

- Resolución 001043 del 3 de abril de 2006, por la cual se establecen las condiciones que deben cumplir los prestadores de servicios de salud para habilitar sus servicios e implementar el componente de auditoría para el mejoramiento de la calidad de la atención y se dictan otras disposiciones. Anexo técnico 1 y 2. 
- Decreto 1011 del 3 de abril de 2006, por el cual se establece el Sistema Obligatorio de Garantía de Calidad de la Atención de Salud del Sistema General de Seguridad Social en Salud.

- Resolución 2434 del julio 11 de 2006, por la cual se reglamenta la importación de equipo biomédico repotenciado Clases IIb y III.

- Decreto 1030 del 3 de marzo de 2007, por el cual se expide el Reglamento Técnico sobre los requisitos que deben cumplir los dispositivos médicos sobre medida para la salud visual y ocular, y los establecimientos en los que se elaboren y comercialicen dichos insumos, y se dictan otras disposiciones.

- Decreto 4957 del 27 de diciembre de 2007, por el cual se establece un plazo para la obtención del registro sanitario o permiso de comercialización de algunos dispositivos médicos para uso humano y se dictan otras disposiciones.

- Resolución 004816 del 27 de noviembre de 2008, por la cual se reglamenta el Programa Nacional de Tecnovigilancia.

- Decreto 2376 del 1 de julio de 2010,por medio del cual se regula la relación docencia-servicio para los programas de formación del talento humano del área de la salud.

- Norma Técnica de Calidad Iso 9001-2000. Esta norma ha sido traducida por el Grupo de Trabajo "Spanish Translation Task Group" del Comité Técnico ISO/TC 176, Gestión y aseguramiento de la calidad, en el que han participado representantes de los organismos nacionales de normalización y representantes del sector empresarial de los siguientes países: Argentina, Chile, Colombia, Costa Rica, Ecuador, España, Estados Unidos, México, Perú, Uruguay y Venezuela. 


\section{EL INSTRUMENTADOR QUIRÚRGICO Y SU COMPROMISO CON LOS PRINCIPIOS Y VALORES ÉTICOS ${ }^{4}$ Y BIOÉTICOS DE ACUERDO CON LA LEY DE TALENTO HUMANO EN SALUd EN COLOMBIA ${ }^{5}$}

El instrumentador quirúrgico se ha profesionalizado en su saber teniendo en cuenta los avances científicos, y asume cada día el compromiso que posee en la atención de los pacientes quirúrgicos, perfeccionando sus técnicas y conocimientos para brindar al final una mejor atención al paciente. En general, existe un enfoque que busca cuidar y velar por los protocolos que hay que cumplir, por ejemplo: las reglas asépticas que evitan una infección cruzada ${ }^{6}$ y otras necesidades en el ambiente quirúrgico, las cuales los convierten en profesionales con destreza y con mucha solidez científica.

Si bien es cierto que el instrumentador quirúrgico tiene varios perfiles ocupacionales en los que se ha destacado, es en el área asistencial en la que predomina su accionar, teniendo en cuenta que el ambiente hospitalario es uno de los más difíciles, pues es el área de quirófano a donde los pacientes llegan con más necesidades afectivas, sumadas a las físicas propias de su estado. Para cumplir estos objetivos, cada persona que interviene en los procedimientos propios del acto quirúrgico debe poseer conocimientos y entrenamientos adecuados, adquiridos a través de una formación académica.

El instrumentador quirúrgico cumple funciones significativas dentro del equipo quirúrgico, entre ellas: coordinar las áreas de cirugía y centrales de esterilización?, participar activamente en el acto quirúrgico y manejar equipos biomédicos. Teniendo en cuenta el rol y la importancia en el ambiente hospitalario del instrumentador quirúrgico, es pertinente preguntarse: ¿conoce este profesional la Ley 1164 de 2007 en la que se registran los principios, valores, deberes y derechos que debe poseer el talento humano en salud en Colombia? ¿Aplica los principios y valores en los actos en los

4 Jorge Alberto Álvarez-Díaz. ¿Bioética latinoamericana o bioética en Latinoamérica? REVISTA LATINOAMERICANA DE BIOÉTICA 1, 2012. Págs. 10-27.

5 Stephanye Carrillo-González, Jaime Lorduy-Gómez y Ruby Muñoz-Baldiris. Profesional de Instrumentación Quirúrgica frente a la aplicación de los principios y valores bioéticos de acuerdo con la Ley de Talento humano en salud en Colombia. PERSONA Y BIOÉTICA 1. 2018. Págs. 134-147.

6 Luis Delpiano. Infecciones cruzadas en las prácticas de salud ambulatorias. MEDWAVE 6. 2009.

7 Stevenson Marulanda Plata. Sanción presidencial de la Ley de Talento Humano en Salud. REVISTA COLOMBIANA DE CIRUgía 4. 2007. Págs. 190-191. 
que se intervenga a un paciente? En relación con estas preguntas, no existen estudios previos en Colombia relativos a esta profesión, de ahí se deriva el presente acápite.

En Colombia, existen unos principios y valores ${ }^{8}$ que rigen a todo el personal que interviene en la promoción, educación, información, prevención, diagnóstico, tratamiento, rehabilitación y paliación de la enfermedad de todos los habitantes del territorio nacional, dentro de la estructura organizacional de la prestación de los servicios de salud. Para profundizar sobre el tema, es importante partir del concepto de principio (del latín principı̆um): "primer instante del ser de algo, punto que se considera como primero en una extensión o en una cosa, norma o idea fundamental que rige el pensamiento o la conducta".

Los principios, según la Ley de Talento Humano en Salud, artículo 35, se enmarcan en el contexto del cuidado respetuoso de la vida y la dignidad de cada ser humano, y en la promoción de su desarrollo existencial, procurando su integridad física, genética, funcional, psicológica, social, cultural y espiritual, sin distinciones de edad, credo, sexo, raza, nacionalidad, lengua, cultura, condición socioeconómica e ideología política, y de un medio ambiente sano. A continuación, se parafrasean los principios aplicados en el quehacer del instrumentador quirúrgico en aras de buscar aplicabilidad:

- Veracidad: orienta el desempeño del instrumentador quirúrgico desde una comunión entre lo que es, piensa, dice y hace, en referencia al trato con las personas en el ejercicio de su profesión.

- Autonomía: es la capacidad que tiene el instrumentador quirúrgico para deliberar, decidir y actuar, con base en un conocimiento científico, a favor de los demás.

- Igualdad: el instrumentador quirúrgico reconoce el mismo derecho para todos los que se benefician de su servicio de recibir una adecuada atención en salud, acorde con las necesidades de cada ser humano.

- Beneficencia: en el ejercicio de su profesión, el instrumentador quirúrgico debe hacer lo que conviene a cada ser humano, respetando los derechos de los demás y procurando que el beneficio para el paciente sea más abundante y menos demandante de esfuerzos en términos de riesgos y costos. Debe respetar de modo especial los derechos de grupos vulnerables limitados en el ejercicio de su autonomía.

8 Lizandro Alfonso Cabrera-Suárez. El significado real de que Colombia sea un Estado Social de Derecho. DIXI 27, 2018. 
- No maleficencia: orienta el desempeño del instrumentador hacia actos que, aunque no beneficien, puedan evitar daño, tratando siempre de defender los mejores intereses del paciente.

- Mal menor: en el ejercicio de la profesión, ante una situación en la que hay que actuar sin dilación, el instrumentador quirúrgico deberá elegir el menor mal, evitando transgredir el derecho a la integridad y las posibles consecuencias que se deriven de no actuar.

- De totalidad: en el ejercicio profesional, el instrumentador quirúrgico participa en actos en los que se hace necesaria la extirpación de una parte del cuerpo que cause daño al organismo, siempre y cuando se apliquen las condiciones para la supresión del miembro y en beneficio de la conservación total del individuo.

- De doble efecto: es éticamente aceptable participar en actos en los que la acción en sí misma sea buena o indiferente, y tenga un doble efecto (uno bueno y uno malo), siempre y cuando: el efecto bueno sea inmediato, el fin de quien realiza la acción sea honesto y exista una causa proporcionada para permitir el efecto malo.

- Valores: el ejercicio de la profesión de Instrumentación Quirúrgica se debe realizar teniendo en cuenta los siguientes valores establecidos en el artículo 36 de la Ley 1164 de $2007^{\circ}$, aplicados a las otras personas, la comunidad, la profesión y las instituciones. Sirven de fundamento a las disposiciones sobre ética profesional del instrumentador quirúrgico.

- Humanidad: el instrumentador quirúrgico debe dar un trato humano a cada paciente de acuerdo con una jerarquía razonablemente sustentada en sus necesidades biológicas, psicológicas, sociales y espirituales.

- Dignidad: el profesional de instrumentación quirúrgica debe reconocer la dignidad de cada ser humano, entendida como la mayor excelencia entre los seres vivos, por lo que en su actuar no participa en ninguna forma de

9 Esta ley se justifica con base en la ausencia de integración intersectorial para el desarrollo de políticas y desarrollo de recurso humano en salud. La carencia de un organismo rector, por lo que no hay planeación, ni control en la formación de los trabajadores de la salud. Por la incoherencia entre la formación del recurso humano y las necesidades de nuestra población. Para incentivar la investigación y educación continua. Y con la necesidad de corregir la posición dominante de las EPS e IPS sobre la autonomía profesional médica. Tienen como objetivos: Articular los actores que intervienen en la: formación, vigilancia y control del ejercicio, el desempeño y la ética de los recursos humanos del área de la salud y garantizar calidad en la formación en salud y escenarios de prácticas, acorde con los cambios académicos y científicos. Se crean nuevos organismos como: el Consejo Nacional de Recursos Humanos Salud, el Observatorio de Recursos Humanos en Salud y los Colegios Profesionales. 
maltrato o discriminación, antes bien, promueve al ser humano dentro de las características especiales, tratándolo como fin, nunca como un medio.

- Responsabilidad: se debe analizar, dar razón y asumir las consecuencias de las propias acciones u omisiones en lo referente al ejercicio de la profesión.

- Prudencia: el instrumentador quirúrgico debe tener sensatez en la conducta práctica; debe realizar una valoración acertada de los medios y de los fines de cada acto; ponderar previamente el fin que desea alcanzar, cuáles son sus consecuencias positivas y negativas para sí mismo, para los pacientes, el equipo de trabajo, y cuáles son los medios y el momento más adecuado para alcanzarlos.

- Secreto: el instrumentador quirúrgico debe salvaguardar la confidencialidad en todo lo que viere u oyere en el ámbito donde se desenvuelve; de igual forma, debe evidenciar confiabilidad y credibilidad en el cumplimiento de los compromisos que adquiere en el ejercicio de su profesión.

\section{Conclusiones}

La Ley 1164, en su capítulo VI, establece parámetros generales que unifican los principios, valores, deberes y derechos del personal responsable que interviene y acompaña a los pacientes, y hace referencia a la caracterización del talento humano en salud. Esta norma aporta y aclara la forma como se deben dar las relaciones entre personal de salud, paciente y familia no solo en el ámbito clínico, sino también en el afectivo (amabilidad, comunicación). Puesta en escena la percepción de principios y valores en los instrumentadores quirúrgicos encuestados, se evidenció que la gran mayoría de ellos, en su quehacer profesional, aplican los principios y valores, aunque muchos desconocen su nombre o definición, entre ellos el mal menor, la no maleficencia, la totalidad y la causa de doble efecto.

Los instrumentadores quirúrgicos respetan las creencias de los pacientes en relación con los procedimientos a realizar, e incluso intervienen cuando un paciente es discriminado, maltratado e instrumentalizado, lo cual es muy importante porque da evidencia de la integridad de su trabajo.

Los instrumentadores no tienen preferencias en la atención de pacientes según sus características (niños, pacientes terminales o en estado crítico); estas, analizadas desde el punto de vista ético, son adecuadas y entrarían en el principio de beneficencia. Sin embargo, hay un porcentaje mínimo pero atrayente que marca la preferencia cuando se trata de un familiar o un conocido; ante este grupo sería adecuado trabajar y reafirmar la escala de principios y valores profesionales, pues se sesga la atención y 
se viola el principio de la igualdad, el cual busca brindar el mismo cuidado a cada uno de los pacientes atendidos.

Se propone ahondar más, desde la academia, en el fortalecimiento de estos temas, ya que sería oportuno afirmar los principios y valores en el profesional de la salud para que este tenga una respuesta acorde con la demanda de atención de los pacientes no solo en la resolución de los aspectos quirúrgicos, sino también en su estado emocional, trascendiendo a un nivel integral y de mayor cuidado para que la atención oportuna y la comunicación personalizada se den en el tiempo de la cirugía y desde el pre y en el posquirúrgico.

Se debe insistir en el respeto y el valor de la vida humana en todas sus dimensiones, con la necesidad de brindar un toque humanizado que traspase la barrera de ese atuendo quirúrgico y logre transmitir interés genuino por el paciente y respeto por su condición, para así seguir formando generaciones de profesionales con alto sentido ético y vocación como "parte integral del equipo de salud"

\section{REFERENCIAS}

Carmen Luz Pretelt de Pretelt. Diplomacia educativa, programa de apoyo a la calidad de la educación superior colombiana. CONGRESO CONVERGENTE DE EDUCACIón SUPERIOR. Veracruz, México. Julio de 2001.

Congreso de la República de Colombia. Ley 30 de 1992. Por la cual se organiza el servicio público de la Educación Superior (M.P. José Gregorio Hernández; diciembre 28 de 1992). DO 40.700.

Congreso de la República de Colombia. Ley 784 de 2002. Por medio de la cual se reforma la Ley 6 del 14 de enero de 1982 (M.P. José Gregorio Hernández; diciembre 23 de 2002). DO 45.046.

Congreso de la República de Colombia. Ley 1438 de 2011. Por medio de la cual se reforma el Sistema General de Seguridad Social en Salud y se dictan otras disposiciones (M.P. José Gregorio Hernández; enero 19 de 2011). Do 47.957.

Daniel Bogoya Maldonado. Proyecto sobre EVALUACIÓn POR COMPETENCIAS. MEMORIAS DEL TALLER SOBRe EVAluación de COMPETEnCIAS BÁsICAS. Bogotá: Editorial Universidad Nacional de Colombia. (1999).

Julio León Trejos. La salud familiar. Un enfoque de atención integral en el contexto de la seguridad social. VI Congreso Colombiano de SALud Familiar. Medellín. 2002. 
Jorge Alberto Álvarez-Díaz. ¿Bioética latinoamericana o bioética en Latinoamérica? REVISTA LATINOAMERICANA DE BIoÉTICA 1, 2012. Págs. 10-27.

Lizandro Alfonso Cabrera-Suárez. El significado real de que Colombia sea un Estado Social de Derecho. DIXI 27, 2018.

Luis Delpiano. Infecciones cruzadas en las prácticas de salud ambulatorias. Medwave 6. 2009.

María Cristina Torrado Pacheco. El DeSARRollo de LAS Competencias: UnA PROPUESTA PARA LA Educación Colombiana. Memorias del taller sobre EVAluación de COMPETENCIAS BÁsicas. Bogotá: Editorial Universidad Nacional de Colombia. (1999).

Ministerio de Educación Nacional. Resolución 2772 de 2003. Por la cual se definen las características específicas de calidad para los programas de pregrado en Ciencias de la Salud (noviembre 13 de 2003).

Ministerio de la Protección Social. República de Colombia. Resolución 02183 de 2004. Por la cual se adopta el Manual de Buenas Prácticas de Esterilización para Prestadores de Servicios de Salud (M.P. Diego Palacio Betancourt; julio 9 de 2004)

Ministerio de la Protección Social. República de Colombia. Resolución 06394 de 2004. Por medio de la cual se adopta el manual de procedimientos del Sistema de Cadena de Custodia para el Sistema Penal Acusatorio (M.P. Alejandro Martínez Caballero; diciembre 31 de 2004).

Ministerio de la Protección Social. República de Colombia. Decreto 1011 de 2006. Por el cual se establece el Sistema Obligatorio de Garantía de Calidad de la Atención de Salud del Sistema General de Seguridad Social en Salud (M.P. Diego Palacio Betancourt; abril 3 de 2006).

Ministerio de la Protección Social. República de Colombia. Decreto 4957 de 2007. Por el cual se establece un plazo para la obtención del registro sanitario o permiso de comercialización de algunos dispositivos médicos para uso humano y se dictan otras disposiciones (diciembre 27 de 2007).

Ministerio de la Protección Social. República de Colombia. Resolución 4816 de 2008. Por la cual se reglamenta el Programa Nacional de Tecnovigilancia (noviembre 27 de 2008).

Renato Ortiz. MundiAlizACIÓn y CULTURA. Buenos Aires: Alianza Editorial. (1996). 
14 Aspectos legales de la instrumentación quirúrgica en Colombia

Stephanye Carrillo-González, Jaime Lorduy-Gómez y Ruby Muñoz-Baldiris. Profesional de Instrumentación Quirúrgica frente a la aplicación de los principios y valores bioéticos de acuerdo con la Ley de Talento humano en salud en Colombia. Persona y BioÉtica 1. 2018. Págs. 134-147.

Stevenson Marulanda Plata. Sanción presidencial de la Ley de Talento Humano en Salud. RevISTA Colombiana de Cirugía 4. 2007. Págs. 190-191. 